\title{
Investigation principles of creation of nanosecond laser driver with operating frequency up to $10 \mathrm{kHz}$
}

\author{
Valery Zemlyakov, \\ National Research University of \\ Electronic Technology-MIET \\ Zelenograd, Moscow, Russia \\ vzml@ rambler.ru \\ Alexandr Fotiadi \\ Peter the Great St. Petersburg \\ Polytechnic University \\ St.-Petersburg, Russia \\ fotiadi@rphf.spbstu.ru
}

\author{
Vladimir Egorkin \\ National Research University of \\ Electronic Technology-MIET \\ Zelenograd, Moscow, Russia \\ egorkin@qdn.miee.ru \\ Sergey Vainshtein \\ CAS Group \\ University of Oulu \\ Oulu, Finland \\ $\underline{\text { vais@ee.oulu.fi }}$
}

\author{
Alexey Filimonov \\ Peter the Great St. Petersburg \\ Polytechnic University \\ St.-Petersburg, Russia \\ filimonov@rphf.spbstu.ru
}

The aim of the work is to create a miniature pulse optical lidar on an infrared laser diode by means of highly effective avalanche switching in bipolar silicon transistors. Confident operation of the optical switch board at a frequency of $10 \mathrm{kHz}$ with a dynamic charging circuit has been achieved. The ways of increasing the radiation power, increasing the repetition rate of pulses (necessary to improve the accuracy of the lidar) are considered.

Keywords. Optical radars, high-speed switching, avalanche drivers, miniature assembly, peak power.

\section{INTRODUCTION}

In modern long-range optical lidar operating in the near infrared range, transmitters with pulse modulation on laser diodes are used, capable of emitting optical pulses with a peak power of up to 40 watts and lasting 3-10 ns. The term Light Detection and Ranging (LIDAR) refers to ranging systems operating in the optical range and using a gas laser or laser diode as a radiation source. For the pumping of high-power broadband laser diodes used in laser lidars and other systems, it is necessary to generate current pulses with duration of several nanoseconds and amplitude of $\sim 10$ $100 \mathrm{~A}[1,2]$. In our opinion, the best available solution to this problem is associated with the use of high-voltage $(\sim$ $300 \mathrm{~V}$ ) avalanche transistors [3] for optical pulses of 3-10 ns duration from laser diodes of $10-100 \mathrm{~W}$ power. The main problem is nanosecond high-current drivers [4, 5]. Highcurrent (10 - 100 A) drivers of the nanosecond, subnanosecond and even picosecond range are on the market (http://www.fidtechnology.com). However, the high price and large size of the available design solutions make their use in real conditions very difficult. Earlier we discussed the design of a miniature nanosecond emitter for optical radar $[6,7]$. The schematic diagram of the current driver is shown in Fig. 1. When measuring the distance to the object, there is a scatter in the results of single measurements and an accurate determination of the distance requires averaging the results. Accuracy increases as the square root of the number of measurements. Accordingly, the operation of the lidar in real time requires a sufficiently high frequency of repetition of measurements. For example, at a frequency of $1 \mathrm{kHz}$, the accuracy of the measurement improves by about thirty times with a measurement time of $1 \mathrm{~s}$, and $10 \mathrm{kHz}$ will provide a hundredfold improvement in the accuracy of the measurements. The main factor limiting the frequency of operation of the avalanche switch is that when switching there is a highly localized heating region and the permissible frequency of repetition of pulses is determined by the time of thermal diffusion from this micron region to the heat sink. In the previously developed modules [6], this time is of the order of $1 \mathrm{~ms}$ and the guaranteed frequency of the module is no more than $0.5 \mathrm{kHz}$. The goal of this work is to analyze main problems to be solved for development miniature low-cost, high-power nanosecond transmitter optimized for long-distance decimeter-precision lidar.

\section{DRIVING CIRCUIT AND A ROLE OF EACH COMPONENT}

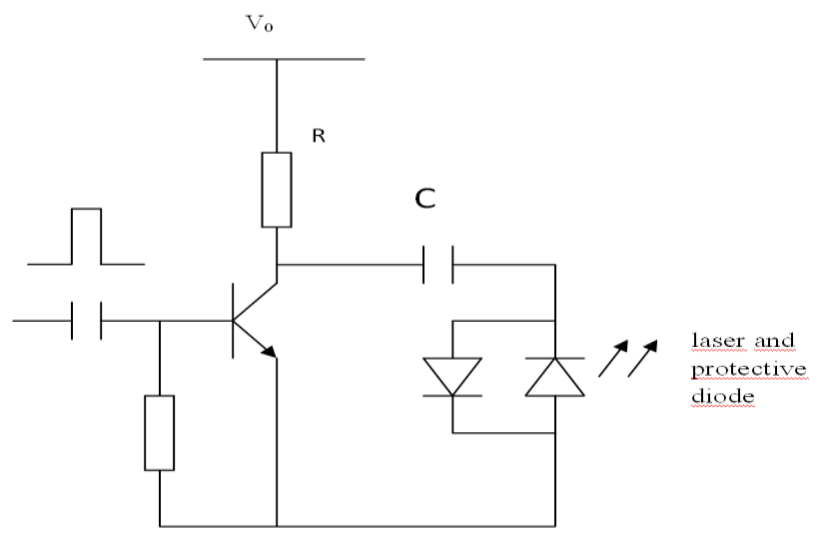

Fig. 1. The current driver is represented by a bipolar transistor operating in the avalanche breakdown mode; the capacitor $\mathrm{C}$ accumulates energy between the pulses (is charged) and discharges through the load resistor $\mathrm{R}$.

\section{RESULTS AND DISCUSSIONS}

In Fig. 2 shows the assembly of a switch based on the above requirements using transistors, laser diodes and a surface mounted chip capacitor. The assembly is performed proceeding from the requirements of the minimum possible distance between the emitter of the transistor and the laser diode to provide the minimum parasitic inductance limiting 
the switching time. To do this, for example, the transistor is mounted on a capacitor, the laser diode - on an aluminumnitride board with the minimum distance between them.

It is established that the main factor limiting the frequency range of the avalanche optical switch operation is the mechanism of high-frequency degradation. Immediately after a single switching, the temperature in the hot region (a cross-section of several $\mu \mathrm{m}$ ) located at the collectorsubcollector boundary reaches $700-800 \mathrm{~K}$. Cooling through a $200 \mu \mathrm{m}$ substrate occurs with a characteristic thermal diffusion time of several tenths of a millisecond $(\sim 0.4 \mathrm{~ms})$. This means that a sufficiently complete cooling, which does not allow the gradual accumulation of heat from pulse to pulse, requires several such time constants $(>1 \mathrm{~ms})$, and therefore $1 \mathrm{kHz}$ is the upper limit of the permissible range of operating frequencies of the radiator. If the next impulse comes earlier than several thermal diffusion time constants, the heat summation in the breakdown region begins, the heat accumulation in the hot region, which after some time (of the order of $10^{5}$ pulses) brings the temperature in this region to the thermal destruction of silicon near the collector-subcollector boundary.

After switching the device, the voltage on it will begin to increase from almost zero, tending to reach the power supply voltage in ms or somewhat earlier. This means that on a still unheated device, the voltage begins to be applied to the "spreading" in all directions in time and gradually cooling the hot region, while the carriers in the conducting channel have not yet recombined. Consequently, the additional heating of the entire channel will begin, preventing the full cooling of the hot area. With a certain set of parameters, this process will lead to the formation of a heat cord, and that, in turn, destroys the device.

Note that the maximum temperature is expected near the boundary of the $\mathrm{n}^{0}-\mathrm{n}^{+}$collector, leading first of all to destruction of the reservoir layer somewhat remote from the p-n junction, and one can expect not a full transistor shortcircuit, but a decrease in the breakdown voltage to a value determined by the thickness of the undamaged part n0 collector layer.

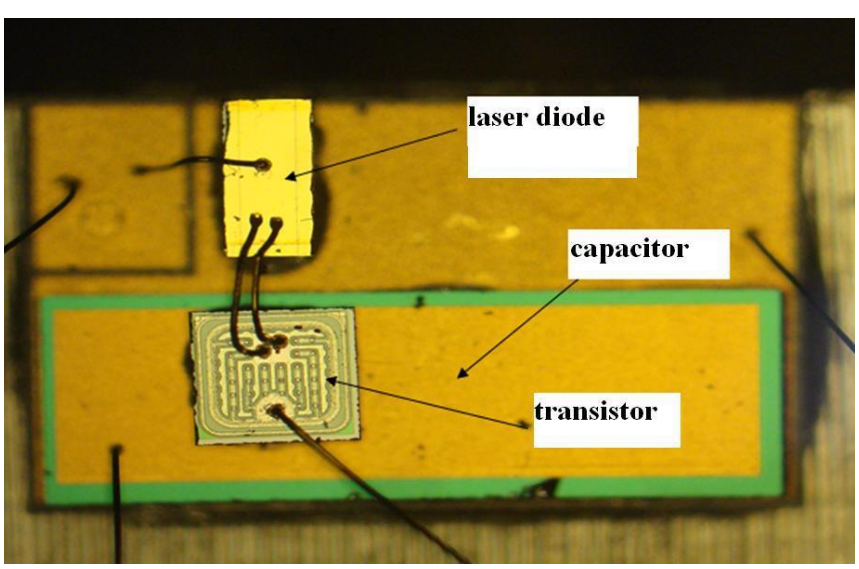

Fig. 2. Assembly of a switch with the installation of a transistor on a capacitor to ensure a minimum parasitic inductance.
This was observed in failed transistors with increasing frequency, the breakdown voltage of the base-collector decreases from $160 \mathrm{~V}$ to $40 \mathrm{~V}$, since the thickness of the remaining weakly doped layer of the collector decreases several times.

Since at present it is not possible to obtain crystals of a transistor with a thinned substrate, in this work the problem of the reliability of the operation of the switch at frequencies up to $10 \mathrm{kHz}$ is solved by other methods. Namely, an additional mechanism for heating the hot zone and the switching channel between nanosecond pulses is eliminated. As already mentioned above, to charge the capacitance, the transistor is connected to a power supply of the order of hundreds of volts through some resistance R (of the order of several tens of $\mathrm{kOhm}$ ), and the still hot region and avalanche current channel start to be heated by the power, determined by the product of the current (decreasing in time from V/R to zero, where $\mathrm{V}$ is the voltage of the power supply) to the voltage (increasing from zero to $\mathrm{V}$ ).

The current flows due to the fact that the carriers in the switching channel have not yet died out by the time when the voltage on the structure has started to increase, and in addition the carriers created by thermal generation in the hot zone take place. To eliminate this additional warm-up, we did the following. The circuit-breaker transistor is switched off from the voltage source before the switching moment, and after a nanosecond current pulse, spherical spreading of heat from the hot region and extinction of nonequilibrium carriers begin. As a result, before the next trigger pulse came, all the media became extinct, and the hot zone managed to cool down significantly (as far as thermal diffusion through the substrate allowed).

After that, the capacitance connected to the collector of the transistor is charged by the active charging circuit to the voltage $\mathrm{V}$, charging is turned off, and the next start of the avalanche transistor occurs. This approach does not, of course, eliminate the problem of cooling the hot area through the substrate, but it eliminates the very undesirable mechanism of additional heating, which has made it possible to increase the operating frequency by almost an order of magnitude.

\section{CONCLUSION}

It was experimentally established that at an operating voltage of $140 \mathrm{~V}$, an optical pulse with a power $50 \mathrm{~W}$ with duration of $1.87 \mathrm{~ns}$ was obtained. Confident operation of the optical switch board at a frequency of $10 \mathrm{kHz}$ has been achieved with the use of original capacitors with a dynamic charging circuit.

A further increase in the frequency is possible provided the thickness of the transistor crystal is reduced. Reducing the thickness leads to a quadratic dependence of the working frequency of the switching of the optical driver, that is, a thinning of the substrate by two to three times would increase the frequency by a factor of four to nine, and a decrease in the distance from the $n^{0}-n^{+}$boundary of the collector to the heat removal by special technological 
methods to $\sim 10 \mu \mathrm{m}$ could be possible to increase the operating frequency to at least $0.1-1 \mathrm{MHz}$.

\section{ACKNOWLEDGEMENTS}

This work was performed under the government order of the Ministry of Education and Science of RF, project \# 11.5861.2017.

\section{REFERENCES}

1. A. Biernat, G. Kompa "Powerful picosecond laser pulses enabling high - resolution pulsed laser radar", $J$. Optics, V. 29, pp.225-228, 1998.

2. A. Kilpela, J. Kostamovaara "Laser pulser for a timeof-flight laser radar", Rev. Sci. Instr., V.68, pp. 2253 2258, 1997.

3. S. N. Vainshtein, G. Duan, A. V. Filimonov, J. T. Kostamovaara "Switching mechanisms triggered by a collector voltage ramp in avalanche transistors with short-connected base and emitter", IEEE Transactions on Electron Devices. 2016, Vol. 63, Iss. 8, P. $3044-$ 3048

4. W. B. Herden, "Application of avalanche transistors to circuits with a long mean time to failure", IEEE Trans. Instrum. Meas., vol. IM-25, no. 2, 740 pp. 152-160, 1976.

5. J.M.T. Huikari, E.A. Avrutin, B.S. Ryvkin, J.J. Nissinen, and J.T. Kostamovaara, "High-Energy picosecond pulse generation by gain switching in asymmetric waveguide structure multiple quantum well lasers", IEEE J. Sel. Topics Quantum Electron., vol. 21, no. 6, 2015, Art. no. 7621501206

6. Filimonov A.V., Zemlyakov V.E., Egorkin V.I., Maslevtsov A.V., Wurz M.C., Vainshtein S.N. "Nanosecond miniature transmitters for pulsed optical radars", Lecture Notes in Computer Science. Vol. 10531 LNCS, 2017, P. 490-497

7. S. Vainshtein, V. Zemlyakov, V. Egorkin, A. Maslevtsov, A. Filimonov. "Miniature high-power nanosecond laser diode transmitters using the simplest possible avalanche drivers", IEEE Transactions on Power Electronics. $2018 . \quad$ DOI: 10.1109/TPEL.2018.2853563 